\title{
Assessment of Caenorhabditis elegans as a model in Haemonchus contortus vaccine research
}

\author{
P. Geldhof ${ }^{\mathrm{a}, \mathrm{b}}$, D. Clark ${ }^{\mathrm{a}}$, C. Molloy ${ }^{\mathrm{a}}$, D.P. Knox ${ }^{\mathrm{a}, *}$ \\ a Moredun Research Institute, Penicuik EH26 OPZ, UK \\ ${ }^{\mathrm{b}}$ Laboratory for Parasitology, Faculty of Veterinary Medicine, Ghent University, Salisburylaan 133, Belgium \\ Received 20 November 2006; received in revised form 12 December 2006; accepted 13 December 2006 \\ Available online 20 December 2006
}

Keywords: Caenorhabditis elegans; Parasite; Model; Vaccine; RNAi; Antibody

Over the past decade Caenorhabditis elegans has become a popular model organism for parasitic nematode research and many examples have been published to illustrate its use [1]. It has especially been useful for the research on mechanisms of action of anthelmintics and on anthelmintic resistance [2]. However, its validity in parasite vaccine research is less clear. It has been suggested that studying genes in C. elegans by RNA interference might help to identify new vaccine targets $[3,4]$. However, as far as we know, this approach has not yet resulted in the identification of new protective antigens. In contrast, the outcome of a recent study actually questions the utility of this approach in vaccine research. C. elegans was used to investigate the function of intestinal cathepsin B-like proteinases in nematodes [5]. These gut expressed enzymes have proven to be effective targets for vaccination against Haemonchus contortus and Ancylostoma caninum, resulting in lower faecal egg output and worm burdens [6,7]. Surprisingly, simultaneous RNAi of the predominant intestinal cathepsin B enzymes in adult $C$. elegans did not result in any visible phenotype despite the complete abolishment of all cysteine proteinase activity, detectable by substrate gel analysis of worm extracts. [5]. One possibility is that the expected phenotype was masked by the activity of other proteinases which could compensate for a loss of digestive function.

Here, in the first instance, homologues of the most established $H$. contortus digestive enzyme vaccine candidates were targeted simultaneously in $C$. elegans by RNAi. These antigens are the metallopeptidase III and pepsin of the H-gal-GP complex, the $\mathrm{H} 11$ aminopeptidase and the cysteine proteinases

\footnotetext{
* Corresponding author. Tel.: +44 131445 5111; fax: +44 1314456111.

E-mail address: david.knox@moredun.ac.uk (D.P. Knox).
}

[8]. These intestinal antigens induce high levels of protection in sheep against a $H$. contortus challenge infection, reducing faecal egg counts by $77-90 \%$ and worm burdens by $47-78 \%$.

All members in C. elegans of the metallopeptidase (peptidase family M13), pepsin (peptidase family A1) and aminopeptidase (peptidase family M1) protein families were identified in the Wormbase database (http://www.wormbase.org) and subsequently used in a phylogenetic analysis with the $H$. contortus proteins using ClustalX [9] and Treeview [10]. Two metallopeptidases (CE14174, CE11095), 1 pepsin (CE16843) and 1 aminopeptidase (CE21162) were identified which clustered together with the $H$. contortus proteins (results not shown). Sequence similarity between the $C$. elegans and $H$. contortus proteins were 20 and $42 \%$ for CE14174 and CE11095, 40\% for CE16843 and 39\% for CE21162. The homologues of the H. contortus intestinal cysteine proteinases were previously identified [5].

RNA interference experiments were set-up to knock down the expression of the metallopeptidases, the pepsin, the aminopeptidase and the CPR 4 cysteine proteinase both individually and simultaneously. The CPR4 cysteine proteinase was chosen because it was shown previously [5] that RNAi directed at this transcript resulted in the complete abolishment of all cysteine proteinase activity in the worm [5]. The double-stranded RNA for each gene was produced and RNAi conducted using methods described previously [5,11]. The primer sequences used to amplify and clone each gene can be obtained from the authors. Larvae soaked in PBS or dsRNA for a $H$. contortus specific superoxide dismutase [5] served as controls. After $24 \mathrm{~h}$ of soaking, the larvae were transferred to NGM plates seeded with OP50 Escherichia coli. Worms were visually examined over a period of 5 days for phenotypic effects such as growth, development and movement. A possible effect on reproduction 
was checked by transferring 10 adult hermaphrodites to fresh NGM plates ( 2 worms per plate). Eggs were counted on each plate after $24 \mathrm{~h}$. An in vivo feeding assay with FITC-labelled bacteria [5] was used to visualize possible effects on worm feeding and Nile Red staining was used as a measure of food digestion and nutrition in the worms [12]. RNAi treated worms, fed on Nile Red, were examined under a fluorescent microscope with an excitation filter of 515-560 nm and an emission filter of $590 \mathrm{~nm}$. The absence of the target transcripts following RNAi treatment was confirmed by gene-specific semiquantitative RT-PCRs using the Superscript One-Step RT-PCR system (Invitrogen) on RNA from 15 adult hermaphrodites $24 \mathrm{~h}$ post-RNAi treatment [11]. Transcript levels of a non-target gene (f54d5.3) were measured as a control for the specificity of the treatment [5]. The transcript levels of a heat shock protein $(h s p 16-1)$ were measured to monitor stress in the worms [13].

Worms treated with individual dsRNA for the two metallopeptidases and the CPR- 4 cysteine proteinase showed almost complete gene silencing for the target genes, as judged by RTPCR (results not shown). However, there were no detectable phenotypes (results not shown). RNA interference experiments for the aminopeptidase, on the other hand, did not result in specific knock down in the expression of this gene despite different dsRNA constructs being tested in repeated experiments, without any success. RT-PCRs on RNA samples from different life stages of $C$. elegans showed that the pepsin homologue (no. CE16843) was not expressed in the adult hermaphrodite life stage (results not shown). Therefore, the aminopeptidase and the pepsin homologue were not included in the combinatorial RNAi experiment.
Worms treated with a mixture of dsRNA to the two metallopeptidases (CE11095, CE14174) and the CPR4 cysteine proteinase showed a significant knock down in the expression of each target gene, compared to expression levels in negative control worms and worms treated with control dsRNA (Fig. 1, panel A). The expression levels of the control gene ( $554 d 5.3)$ and the hsp 16-1 gene were not affected, indicating that the gene knock down was specific and did not induce a major stress response. The RNAi treated worms did not show any obvious phenotypic abnormalities. Egg output (Fig. 1, panel B) was increased by 21 $\%$ in the RNAi treated worms compared to the dsRNA control worms. The in vivo feeding and nutrition assays indicated that there was no effect in the RNAi treated worms on food uptake and digestion. Typical results for the Nile Red staining are shown in Fig. 1 panel C. The RNAi treated worms (MEP + CPR4) showed similar staining throughout the intestine compared to control worms.

Nowell et al. [13] used C. elegans to monitor immunological stress in nematodes by incubating $C$. elegans in antibodies directed against the worm cuticle to mimic an immune attack. The results indicated that the physical binding of antibody induced a stress response with a significant reduction in growth and fecundity of the adult worms. At the moment, the best working native vaccines against $H$. contortus are all based on gut expressed proteins [8] and, more importantly, antibody is thought to mediate this protective immunity by blocking or interfering with the digestion within the worm. One possibility is that this "physical" effect acts synergistically with specific enzyme inhibition to impair worm digestion and that RNAi targeting the C. elegans homologues does not induce a phenotype because the physical effect is absent. To test this hypothesis, we targeted the

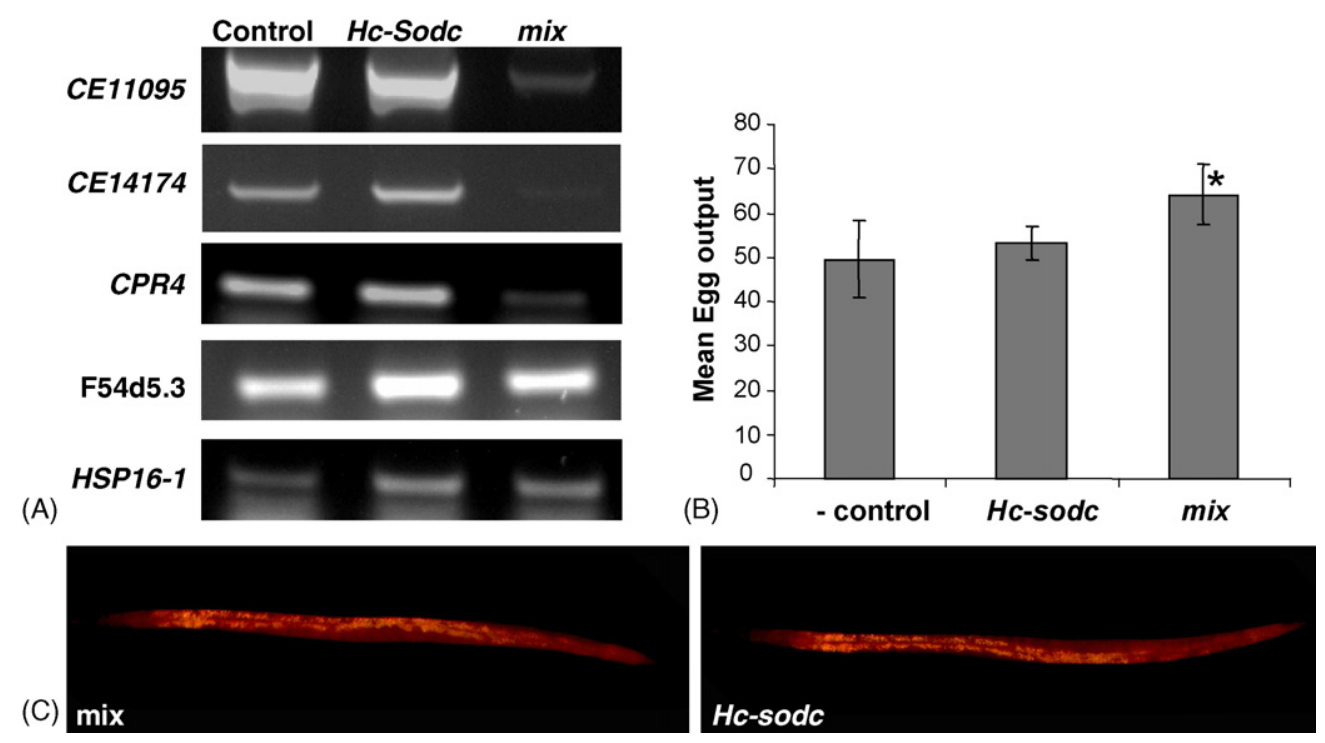

Fig. 1. Adult hermaphrodite C. elegans worms were treated with a dsRNA mixture to knock down the expression of two metallopeptidases (CE11095 and CE14174) and a cysteine proteinase (cpr4) and were subsequently checked for effects on feeding, development and reproduction. (A) Loss of CE11095, CE14174 and $c p r 4$ transcripts following RNAi. Semi-quantitative RT-PCRs were carried out on total RNA isolated from 15 wild-type worms (control), 15 worms treated with control dsRNA $(H c-s o d c)$ and 15 worms treated the dsRNA mixture (mix). RT-PCRs for two control genes ( $f 54 d 5.3$ and $h s p 16-1)$ were run to check the quality of the RNA samples. (B) Effect of the RNAi treatment on worm fecundity. The results are shown as mean egg output for two worms after $24 \mathrm{~h}$ on the plate (five replicates). Egg output was increased by $21 \%(P<0.02)$ in the RNAi treated worms compared to the dsRNA control worms. (C) In vivo nutrition assay with Nile Red staining. Nile red is a lipophilic stain and when mixed with $E$. coli $(0.1 \mu \mathrm{g} / \mathrm{ml})$ the worms take up the Nile Red and incorporate it into lipid droplets of the intestinal cells, staining them red under a fluorescent microscope. The RNAi treated worms (mix) showed similar staining throughout the intestine compared to control worms. 
gut expressed proteins in C. elegans with antibodies directed at glycoproteins expressed on the intestine surface [14] and sought effects on feeding, development and reproduction. The antibodies we used in this experiment were purified from pooled sera, obtained either pre- or post-immunisation from sheep vaccinated with $C$. elegans ConA-binding proteins [14], by affinity chromatography over a HiTrap Protein G column (GE Healthcare, UK). This ConA-binding protein fraction contains a complex set of glycoproteins, including cysteine and metallo proteinases (results not shown), purified from the intestine of $C$. elegans. To determine any affect of IgG binding on worm fecundity, a well-based IgG assay was set-up. Two L3/L4 stage C. elegans were transferred to wells containing $90 \mu \mathrm{l}$ PBS (pH 7.4) plus pre- or post-immunisation ConA-binding fraction reactive $\mathrm{IgG}$ at a final dilution of 1:10. Ten replicates of each treatment were set-up. To allow worms to feed, $10 \mu \mathrm{l}$ OP50 strain E. coli from an overnight bacterial culture was added to each well. Following incubation at room temperature for 2 days, worms were killed by addition of $2 \mu \mathrm{l}$ helminthological iodine and the number of L1/L2 stage larvae in each well enumerated. FITC-labelled bacteria and the Nile red staining were used to monitor food uptake and nutrition. Binding of antibody to the intestine was confirmed by transferring 15 worms to NGM agar plates. Following incubation for $1 \mathrm{~h}$, worms were picked and incubated in PBS containing FITC-labelled mouse anti-sheep IgG (Sigma, UK) at a final dilution of 1:50 for $2 \mathrm{~h}$ at room temperature. Worms were subsequently transferred to NGM agar for an hour after which they were transferred into $50 \mu \mathrm{l}$ PBS for analysis under a fluorescent microscope. Stress induced by the treatment was monitored by measuring changes in transcript levels of the C. elegans heat shock protein $h s p 16-1$. This was done by a semi-quantitative one-step RT-PCR on total RNA as described above.

The results from the immunostaining are shown in Fig. 2 panel A. Worms incubated in antibodies purified from immune serum clearly had antibodies bound to their intestinal surface.
Nevertheless, there was no phenotypic difference between worms incubated with and without antibody, in the presence of pre-immune or immune antibodies. Growth, development, movement, reproduction, food uptake and nutrition were not affected. The results for worm fecundity are shown in Fig. 2 panel B. In addition, $h s p 16-1$ transcript levels were not affected in untreated worm and worms incubated in antibodies, both preimmune and immune (results not shown). To assess a potentially synergistic effect of combined RNAi and antibody treatment, experiments were carried out in which worms were pre-treated with a mixture of metallopeptidase and CPR4 or control dsRNA as before. Following RNAi, worms were incubated with either pre- or post-immune IgG as described above. Five replicates of each treatment were set-up. The results for worm fecundity are shown in Fig. 3. No treatment combination had a discernable effect on phenotype (assessed as before) and there was no statistical difference in fecundity between treatments.

The aim of this study was to assess and compare the use of two proposed $C$. elegans-based methods to identify parasite vaccine targets involved in digestion. Neither approach, either alone or in combination, induced any visible phenotypes in the worms. The lack of phenotype might be explained by the fact that the genes targeted in this study are all members of multigene families and it is possible that genes within such families have some functional redundancy. Alternatively, phenotypes might have been become visible if worms were kept in the presence of antibody or dsRNA for longer periods. However, worms treated with dsRNA were already observed for 5 days. During this period there was no sign that the development of the worms stopped or that they were slowly starving, in contrast, the worms were still growing. Finally, the target genes were identified based on their sequence homology and although they are the closest homologues, there is no guarantee that their function is conserved with the $H$. contortus genes. In conclusion, the data presented in this paper clearly infers that both these screening methods
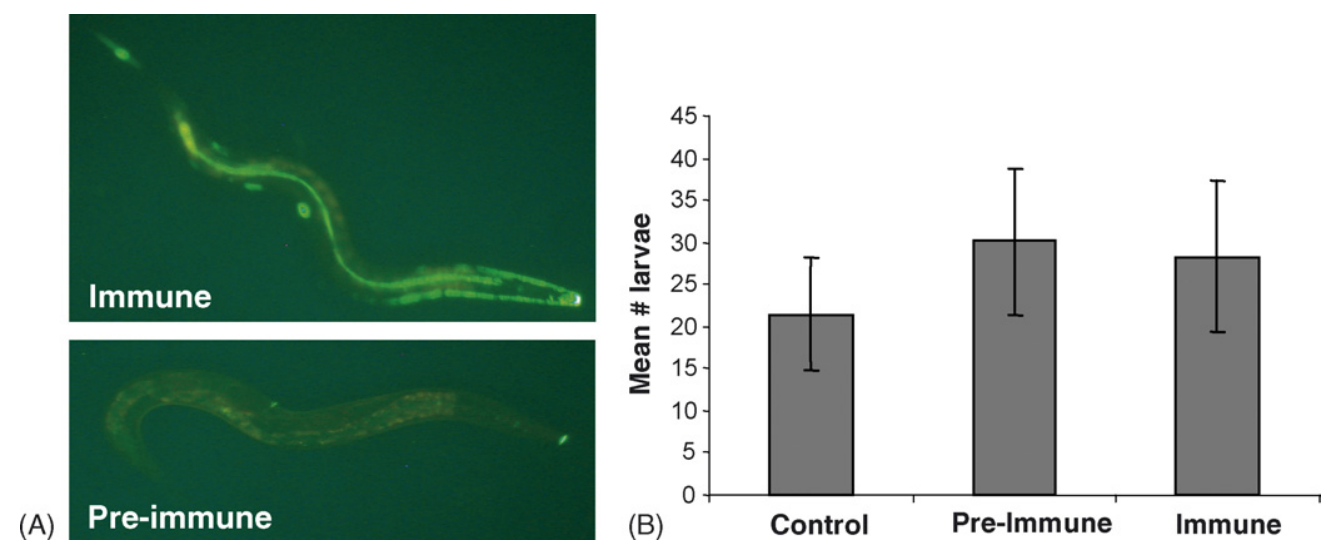

Fig. 2. (A) Detection of antibodies bound to the intestinal surface of hermaphrodite C. elegans worms. Worms were soaked in sheep antibodies raised against gut expressed proteins for 2 days, following this incubation they were transferred to a 1:50 dilution of FITC labelled anti-sheep conjugate for $2 \mathrm{~h}$. Worms were subsequently transferred to NGM agar for an hour after which they were transferred into $50 \mu 1$ PBS for analysis under a fluorescent microscope. Pre-immune serum was used as a negative control. (B) Effect of IgG binding on worm fecundity. Two L3/L4 stage C. elegans were transferred to wells containing 90 $\mu 1$ PBS (pH 7.4) plus pre- or post-immunisation ConA-binding fraction reactive IgG at a final dilution of 1:10. Ten replicates of each treatment were set-up. To allow worms to feed, $10 \mu \mathrm{OP} 50$ strain E. coli from an overnight bacterial culture was added to each well. Following incubation at room temperature for 2 days, worms were killed by addition of $2 \mu 1$ helminthological iodine and the number of L1/L2 stage larvae in each well enumerated. 


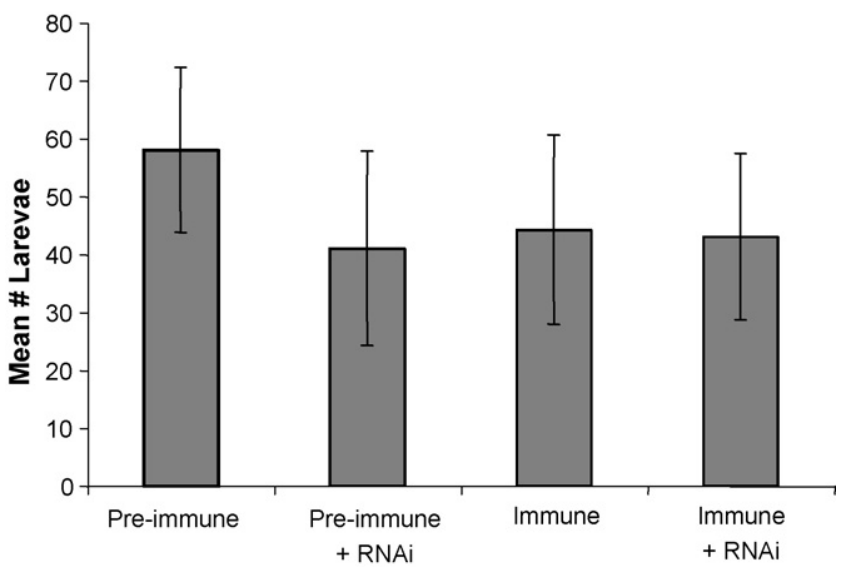

Fig. 3. Effect of combined RNAi and antibody treatment on worm fecundity. Worms underwent RNAi by soaking for $12 \mathrm{~h}$ in the dsRNA mixture for the metallopeptidases and CPR4. These worms were subsequently transferred to wells ( 2 worms/well) containing $90 \mu$ PBS (pH 7.4) plus pre- or post-immunisation ConA-binding fraction reactive $\mathrm{IgG}$ at a final dilution of 1:10. Five replicates of each treatment were set-up. To allow worms to feed, $10 \mu l$ OP50 strain E. coli from an overnight bacterial culture was added to each well. Following incubation at room temperature for 2 days, worms were killed by addition of $2 \mu 1$ helminthological iodine and the number of L1/L2 stage larvae in each well enumerated. Fecundity was compared with worms which did not undergo the RNAi treatment.

would not identify gut expressed proteins as vaccine candidates against $H$. contortus. This, in turn, suggests the need for considerable caution in interpreting $C$. elegans-based data to identify potential parasite vaccine targets.

\section{Acknowledgement}

PG is the recipient of a Marie Curie reintegration grant (no. 028870).

\section{References}

[1] Britton C, Murray L. Using Caenorhabditis elegans for functional analysis of genes of parasitic nematodes. Int J Parasitol 2006;36:651-9.

[2] Geary T, Thompson DP. Caenorhabditis elegans: how good a model for veterinary parasites? Vet Parasitol 2001;101:371-86.

[3] Hashmi S, Tawe W, Lustigman S. Caenorhabditis elegans and the study of gene function in parasites. Trends Parasitol 2001;17:387-93.

[4] Kuwabara PE, Coulson A. RNAi-prospects for a general technique for determining gene function. Parasitol Today 2000;16:347-9.

[5] Geldhof P, Molloy C, Knox DP. Combinatorial RNAi on intestinal cathepsin B-like proteinases in Caenorhabditis elegans questions the perception of their role in nematode biology. Mol Biochem Parasitol 2006;145:128-32.

[6] Redmond D, Knox DP. Protection studies in sheep using affinity-purified and recombinant cysteine proteinases of adult Haemonchus contortus. Vaccine 2004;22:4252-61.

[7] Loukas A, Bethony JM, Williamson AL, et al. Vaccination of dogs with recombinant cysteine protease from the intestine of canine hookworms diminishes the fecundity and growth of worms. J Infect Dis 2004:1952-61.

[8] Knox DP, Redmond DL, Newlands GF, Skuce PJ, Pettit D, Smith D. The nature and prospects for gut membrane proteins as vaccine candidates for Haemonchus contortus and other ruminant trichostrongyloids. Int $\mathbf{J}$ Parasitol 2003;33:1129-37.

[9] Thompson JD, Gibson TJ, Plewnaik F, Jeanmougin F, Higgins DG. The ClustalX windows interface: flexible strategies for multiple sequence alignment aided by quality analysis tools. Nucl Acids Res 1997;25:4876-82.

[10] Page RDM. Treeview: an application to display phylogenetic trees on personal computers. Comput Appl Biosci 1996;12:357-8.

[11] Geldhof P, Whitton C, Gregory WF, Blaxter M, Knox DP. Characterisation of the two most abundant genes in the Haemonchus contortus expressed sequence tag dataset. Int J Parasitol 2005;35:513-22.

[12] Ashrafi K, Chang FY, Watts JL, et al. Genome-wide RNAi analysis of Caenorhabditis elegans fat regulatory genes. Nature 2003;421:268-72.

[13] Nowell MA, Pomerai DIDE, Pritchard DI. Caenorhabditis elegans as a biomonitor for immunological stress in nematodes. Parasite Immunol 1999;21:495-505.

[14] Redmond DL, Geldhof P, Knox DP. Evaluation of Caenorhabditis elegans glycoproteins as protective immunogens against Haemonchus contortus challenge in sheep. Int J Parasitol 2004;34:1347-53. 